\title{
Serum proteomics analysis from rheumatoid arthritis patients receiving combination therapy of imrecoxib, total glucosides of paeony and two DMARDs
}

\section{Haixia Yu}

the First Affiliated Hospital of Anhui Medical University

\section{Xuejing Ma}

the First People's Hospital of Hefei

\section{Xing Fang}

the Second People's Hospital of Hefei

\section{Chunlan Yang}

the First Affiliated Hospital of Anhui Medical University

\section{Xianjun Fang}

the First Affiliated Hospital of Anhui Medical University

Tingting Wang

Durbrain Medical Laboratory, Hangzhou, China

\section{Lanxin Qin}

Durbrain Medical Laboratory, Hangzhou, China

\section{Quan Xia ( $\nabla$ xiaquan2010@163.com )}

the First Affiliated Hospital of Anhui Medical University

\section{Research Article}

Keywords: Rheumatoid arthritis, Combination drug therapy, Proteomics, Imrecoxib, Disease-modifying antirheumatic drugs

Posted Date: March 7th, 2022

DOI: https://doi.org/10.21203/rs.3.rs-1421264/v1

License: (c) (i) This work is licensed under a Creative Commons Attribution 4.0 International License. Read Full License 


\section{Abstract}

BackgroundखRheumatoid arthritis (RA) is a chronic autoimmune disease. Imrecoxib, disease-modifying anti-rheumatic drugs (DMARDs) and total glucosides of paeony (TGPs) are common clinical therapies for RA. However, the exact mechanism of combination therapy for rheumatoid arthritis is still unclear. This study aims to explore the potential mechanism of this combination therapy for RA.

Methods $\mathbb{X}$ Five RA patients who used this combination drug therapy were enrolled. Serum was collected at the first visit and the second visit after 3 months of continuous use of the combination treatment. Labelfree quantitative proteomics was used to perform a comparative analysis of protein expression in serum after depletion of high-abundance proteins. The GO database was used to annotate the functions of differentially expressed proteins (DEPs).

Results】 total of 257 reliable proteins were identified. Six proteins (IGKV3-20, TTR, LRG1, SERPINA3, F10 and NMNAT3) were downregulated, and one protein (HBD) was upregulated after three months of continuous combination drug treatment. Bioinformatics analysis showed that DEPs were primarily involved in various biological processes, such as metabolic processes, cellular processes and biological regulation. The identified DEPs have been reported to be involved in inflammation, cell proliferation, angiogenesis and oxidative stress. Therefore, these proteins have played important roles in RA progression.

ConclusionXThis combination drug therapy could alleviate the symptoms of RA by regulating these DEPs associated with RA in different ways. The DEPs identified may help to predict the treatment outcome of therapy for RA in the future.

\section{Introduction}

Rheumatoid arthritis (RA) is a chronic inflammatory disease predominantly affecting the surrounding joints, with synovitis, cartilage destruction, bone erosion, and disability as its main clinical manifestation $[1,2]$. It is believed that the earlier RA is diagnosed and treated, the better the disease remission and prognosis [3]. To date, even extensive studies have been performed on the pathogenesis of RA, and its specific pathogenesis is still unclear. Clinically, there are many drugs used to alleviate the development of $\mathrm{RA}$, and combination drug therapy with synthetic disease-modifying anti-rheumatic drugs (DMARDs) is a recommended treatment strategy.

DMARDs, including methotrexate (MTX) and leflunomide (LEF), are a class of slowly regulating drugs that can not only improve RA symptoms but also mitigate clinical and radiographic progression [4]. MTX is an immunosuppressant with an anti-inflammatory effect that improves the progression of RA. MTXbased treatment plays an increasingly important role during the early stage of RA treatment $[5,6]$. In the clinical treatment of RA, MTX is often required in combination with other drugs to improve the treatment effect, such as LEF [7, 8]. LEF is also an immunomodulator that can improve the condition of RA [9]. Total glucosides of paeony (TGPs) have the functions of regulating immunity and anti-inflammatory and 
analgesic effects and have become a choice for the treatment of autoimmune diseases in China [10]. Furthermore, TGP has been reported to reduce drug-induced liver injury during combination treatment with LEF and MTX for patients with active RA [11]. RA is an inflammatory disease, and nonsteroidal antiinflammatory drugs (NSAIDs) as the first-line therapy are used to suppress inflammation, which can rapidly relieve the pain and swelling degree [4]. Imrecoxib is a new NSAID that selectively inhibits cyclocxygenase-2 (Cox-2) and is approved for the treatment of osteoarthritis [12]. Compared with monotherapy, the combination of these drugs shows a higher efficacy and lower toxicity in the treatment of RA [13]. The exact mechanism of the combination of these drugs to alleviate the disease remains unclear. Therefore, we studied the mechanism at the protein level to explore whether the combination of drugs achieves the remission of RA through specific proteins.

With the development of advanced mass spectrometers (MS) and the continuous optimization of methods, mass spectrometer-dependent proteomics has gradually become an important and reliable tool to study the mechanism of drugs at the protein level [14]. Therefore, we used label-free quantitative proteomics to screen for differentially expressed proteins (DEPs) in serum after combination drug therapy and serum after 3 months of continued use of combination drug therapy. The gene ontology (GO) annotation analysis of the DEPs was analysed by the PANTHER Classification System. We explored the specific mechanism of the combination therapy by screening for DEPs to better clarify the mechanism of combined drug therapy for RA and to explore a better method for the treatment of RA through these mechanisms.

\section{Materials And Methods}

\section{Patients}

Subjects with RA were recruited from the Department of Rheumatology, the First Affiliated Hospital of Anhui Medical University. Subjects were recruited on the basis of these criteria: (1) conforming to 2010 criteria for RA of the American College of Rheumatology or European League Against Rheumatism; (2) taking a combination of four drugs, imrecoxib $(0.1 \mathrm{~g}$, bid), MTX (10 mg, qw), LEF ( $5 \mathrm{mg}$, qd) and TGP (0.3

$\mathrm{g}$, bid) for more than three months. Serum samples were taken at the first visit and the second visit after 3 months of continuous use of the combination treatment. Samples were divided into two groups: serum samples collected at the first visit (control group) and serum samples collected at the second visit after 3 months (treatment group) (Fig. 1a). Subjects were excluded from these criteria if they (1) had other connective tissue diseases and (2) were pregnant or lactating women. Finally, we collected 5 eligible patients for this study.

\section{Ethical approval}

and sample collection

The study was conducted at the recommendation of the Clinical Medical Research Ethics Committee of the First Affiliated Hospital of Anhui Medical University, and the approval number of ethical reviews is 
PJ2020-03-19. In addition, the subjects were informed of this study. We obtained written informed consent from the participants to publish the material. All work conducted in accordance with the Declaration of Helsinki (1964). Two milliliters of venous blood was collected and centrifuged for $5 \mathrm{~min}$ at $3000 \mathrm{rpm}$, and then the serum was collected and stored at $-80^{\circ} \mathrm{C}$ for further use.

\section{Proteomic analysis}

Extraction and digestion of proteins: Twelve high-abundance proteins in serum, including $₫ 1$-Acid Glycoprotein, \1-Antitrypsin, $₫ 2$-Macroglubulin, Albumin, Apolipoprotein A-I, Apolipoprotein A-II, Fibrinogen, Haptoglobin, IgA, IgG, IgM and Transferrin, were removed by Pierce Top 12 Abundant Protein Depletion Spin Columns (Thermo Fisher Scientific, Massachusetts, USA). Protein concentration was detected by a BCA kit (Beyotime Biotechnology, Shanghai, China). Four times the volume of precooled acetone was added to the protein solution overnight. The above mixed liquid was centrifuged at $10000 \mathrm{~g}$ for $5 \mathrm{~min}$, acetone was poured out, and the precipitate was redissolved in urea and $50 \mathrm{mM}$ ammonium bicarbonate solution. Dithiothreitol $(10 \mathrm{mM})$ and iodoacetamide $(30 \mathrm{mM})$ were added to the protein solution and incubated separately for $15 \mathrm{~min}$. Trypsin (Promega, Wisconsin, USA) was added to the solution at a ratio of $1: 40$ to cleave proteins into peptides at $37^{\circ} \mathrm{C}$ overnight. A ZipTip pipette (Thermo Fisher Scientific, Massachusetts, USA) was used to desalt the lysate. Then, the desalted lysate was freeze-dried.

LC-MS/MS: The dried peptides were redissolved in $0.1 \%$ formic acid and centrifuged at 20,000 $\mathrm{g}$ for 10 min, and the supernatant was injected. Separation was performed by a nano-ESI HPLC system (Dionex, Thermo Fisher Scientific, Massachusetts, USA), which was configured with a C18 column $(5 \mathrm{~mm}, 100 \mathrm{~mm}$ $\times 0.1 \mathrm{~mm}, 300 \mathrm{~A}$ ). Peptides were separated at a flow rate of $300 \mathrm{~nL} / \mathrm{min}$ through the following gradient elution program. The gradient elution was set as follows: $5 \%$ buffer $B$ for 5 min; $5 \%-35 \%$ buffer $B$ for 40 min; $35 \%-90 \%$ buffer B for 5 min; $90 \%$ buffer B for 5 min; $90 \%-5 \%$ buffer B for $1 \mathrm{~min}$; buffer A ( $5 \%$ acetonitrile (ACN), $0.1 \%$ formic acid (FA)) was mobile phase $A$; and buffer $B(95 \% A C N, 0.1 \% F A)$ was mobile phase $B$.

A Q-Exactive Focus Orbitrap MS high-resolution mass spectrometer (Thermo Fisher Scientific, Massachusetts, USA) was used to detect peptides separated in the liquid phase. The operating parameters were set as follows: (1) full MS: 70000 resolution; automatic gain control (AGC) target of 1e6; maximum injection time (MIT) of $50 \mathrm{~ms} ; 350-2000$ (m/z); 20 loop count; NCE 27; (2) dd-MS/MS: 17,500 resolution; AGC target of 1e5; MIT of 50 ms; charge exclusion, exclusion 1, 7, 8, > 8; filter dynamic exclusion duration $20 \mathrm{~s}$; and $1.6 \mathrm{~m} / \mathrm{z}$ isolation window.

The collected mass spectrometry data were analysed with Protein Discoverer (Version 2.4, Thermo Fisher Scientific, Massachusetts, USA) against the human protein database. The false discovery rate was controlled to be under $1 \%$ with $10 \mathrm{ppm}$ mass tolerance for precursor ions and $0.02 \mathrm{Da}$ mass tolerance for product ions. The quantitative data were normalized by the sum of all peptides. The boxplot shows the abundance of all proteins in each sample after normalization.

\section{Bioinformatics analysis}


GO annotation analysis of identified proteins and DEFs was performed using the Panther Classification System (http://www.pantherdb.org/), which includes molecular functions, biological processes and cell components.

\section{Statistical analysis}

The correlation coefficient between each sample was calculated by the Pearson method. $P$ valuevalues were adjusted by Bonferroni correction, and values smaller than 0.05 were considered to represent statistically significant differences. Commonly, Student's t test was applied to compare the distribution of variables between two cohorts. The waterfall displayed all the identified and quantified proteins ranked based on the protein expression level, and the absolute value of fold change was displayed for the differentially expressed proteins with the significantly expressed proteins labeled. To identify DEPs, a $p$ value $\leq 0.05$ and fold change $>1.25$ or $<0.8$ were used as filter thresholds, as shown in volcanoes. Heatmaps and principal component analysis with hierarchical clustering analysis were applied to visualize the characteristics of all DEPs based on their normalized values.

\section{Results}

\section{Clinical characteristics of patients}

The Disease Activity Score in 28 Joints (DAS28) score is now the basis for the treatment of RA, allowing us to evaluate the remission of disease and the effectiveness of current treatment regimens. Rheumatoid factor (RF) can help to diagnose and detect remission of the disease. As shown in Fig. 1b and 1c, all 5 investigated patients presented a significant reduction in the DAS28 and serum RF after three months of continuous use of the combination regimen, including imrecoxib, LEF, TGP and MTX, suggesting the effectiveness of the combination regimen for RA.

\section{Quality control and unsupervised analysis for mass spectrometry data}

First, the result of mass error analysis showed that the mass error was within $10 \mathrm{ppm}$, indicating the high accuracy of the collected data (Fig. 2a). Before differential expression analysis of the sample proteins, we performed quality control analysis by boxplots and principal component analysis (PCA). Boxplot analysis of samples is shown in Fig. 2b. After normalization, the median protein abundance was basically on a horizontal line in each sample. In addition, we performed PCA for the identified DEPs. In Fig. 2c, the PCA results of the two groups showed that the DEPs were segregated into two separate clusters that could distinguish the treatment group from the control group. We performed correlation coefficient analysis for each serum sample by the Pearson correlation coefficient method. From the analysis results, we found that the correlation coefficient between each serum sample was close to 1 , indicating a high correlation coefficient (Fig. 2d).

\section{Quantitative protein detection}


In our study, proteins were quantified and identified in serum collected from subjects before and after three months of combination treatment with imrecoxib, MTX, LEF and TGP by label-free quantitative proteomics. As a result, peptides and 257 proteins were identified. Then, we identified DEPs by standard fold change $>1.25$ or $<0.8$ and $p \leq 0.05$. Compared with the control group, a total of 7 proteins were differentially expressed in the treatment group. Among these DEPs, 6 proteins were downregulated and 1 protein was upregulated after treatment. The details of DEPs are shown in Table 1. Waterfall analysis vividly showed the 7 DEPs with different distributions in all identified proteins (Fig. 3a). The DEPs are also shown in a volcano map (Fig. 3b). Significantly upregulated proteins are shown as red dots, and 6 significantly downregulated proteins are shown as blue dots. Then, these DEPs were used to draw a cluster analysis heatmap, which intuitively reflected expression differences between the two groups (Fig. 3c). The abundance of 7 DEPs in serum is shown in Fig. 4.

Table 1

list of DEPs

\begin{tabular}{|llll|}
\hline Accession & $\begin{array}{l}\text { Gene } \\
\text { name }\end{array}$ & Description & $\begin{array}{l}\text { FC } \\
\text { (Treatment/Control) }\end{array}$ \\
\hline P01619 & IGKV3-20 & Immunoglobulin kappa variable 3-20 & 0.18 \\
\hline P02766 & TTR & Transthyretin & 0.74 \\
\hline P01011 & SERPINA3 & Alpha-1-antichymotrypsin & 0.75 \\
\hline P00742 & F10 & Coagulation factor X & 0.65 \\
\hline D6RHV4 & NMNAT3 & $\begin{array}{l}\text { Nicotinamide/nicotinic acidmononucleotide } \\
\text { adenylyltransferase 3 (Fragment) }\end{array}$ & 0.33 \\
\hline P02750 & LRG1 & Leucine-rich alpha-2-glycoprotein & 0.69 \\
\hline P02042 & HBD & Hemoglobin subunit delta & 6.72 \\
\hline
\end{tabular}

\section{GO annotation analysis}

The molecular functions, biological processes and cell components of the identified proteins are shown in Fig. 5a. The most enriched molecular functions (out of $6 \mathrm{GO}$ terms) were located in catalytic activity, binding and molecular function regulator. The most enriched biological processes (out of $13 \mathrm{GO}$ terms) were located in metabolic process, cellular process and biological regulation. For cellular components, most of the identified proteins were located in cellular anatomical entities (out of $3 \mathrm{GO}$ terms). Next, we carried out GO enrichment analysis of the identified DEPs (Fig. 5b). The molecular functions of these DEPs were molecular function regulators, transporter activity, binding and catalytic activity. For biological processes, DEPs were located in response to stimulus, immune system process, biological regulation, cellular process and metabolic process. For cellular components, these DEPs were located in proteincontaining complexs and intracellular and cellular anatomical entities. 


\section{Discussion}

RA is a chronic autoimmune disease that is characterized by inflammation of the joints and the subsequent destruction of cartilage and erosion of the bone [15]. The exact causes of RA are unclear, but some factors are believed to increase the risk of developing the disease, including genetic, environmental, hormonal and psychological factors [1]. Currently, the main drugs for RA treatment are anti-inflammatory drugs and immunosuppressants. Imrecoxib, a COX-2 enzyme inhibitor, has been proven for osteoarthritis treatment [12]. Immunosuppressants are used to treat RA by suppressing the immune response, including MTX and LEF. TGP is reported to show anti-inflammatory and immunomodulatory effects $[4,10]$. In our study, proteomics analysis was used to explore DEPs after three months of combined treatment with these four drugs to discover the specific mechanism of the combination drug treatment for RA.

In our study, the results showed that 7 proteins were differentially expressed after combination drug treatment for 3 months through label-free quantitative proteomics. Six proteins (IGKV3-20, TTR, LRG1, SERPINA3, F10 and NMNAT3) were downregulated, and one protein (HBD) was upregulated. Four of the DEPs were directly or indirectly involved in the development of RA. These proteins may be the target of drug combination therapy.

GO enrichment analysis in our present study indicates that the DEPs identified herein are related to transporter activity. Transthyretin (TTR), a transporter of thyroxine, was downregulated after combination drug therapy. TTR is a highly conserved homotetrameric protein and is enriched in human plasma and cerebrospinal fluid $[16,17]$. Previous proteomics studies have reported that TTR expression in RA patients was higher than that in healthy people, indicating that TTR might be a potential marker for RA progression $[18,19]$. Reactive oxygen species (ROS), as intracellular signaling molecules, are associated with various inflammatory and chronic joint diseases, including RA [20, 21]. In myeloid cells, TTR treatment increases ROS production, suggesting that TTR may promote the development of RA by affecting the production of ROS [22]. Therefore, combination therapy may improve RA by decreasing TTR.

Leucine-rich a-2-glycoprotein 1 (LRG1), a member of the leucine-rich repeat family of proteins, is reported to be involved in a wide variety of pathophysiological processes, such as angiogenesis, tumor formation and osteoarthritis. A previous study showed that the level of serum LRG1 was increased in RA patients compared with normal controls and decreased by anti-TNF treatment. Moreover, serum LRG1 was positively correlated with C-reactive protein (CRP), erythrocyte sedimentation rate (ESR) and DAS28-CRP score, suggesting that LRG1 might be used as an additional marker for RA [23]. In addition, another study reported that serum LRG1 levels were reduced after treatment with the anti-IL-6 receptor antibody tocilizumab [24]. T cells can differentiate into T helper 17 cells (Th17) or regulatory $T$ cells (Tregs) under different stimulations [25]. The level of Th17 cells is positively associated with the disease activity of RA [26]. Experiments in mice with collagen-induced RA reveal the important role of LRG in RA development and Th17 differentiation. LRG promoted the differentiation of naive CD 4 T cells into Th17 cells by activating the TGF- $\beta$-smad2 pathway [27]. Angiogenesis is a notable characteristic feature of RA. LRG1 
has been found to promote angiogenesis by regulating endothelial cell mitosis [28]. LRG1 may be involved in the process of RA by promoting angiogenesis. Therefore, RA may also be improved by combination therapy by regulating LRG1.

$\mathrm{F} 10$ is vitamin $\mathrm{K}$-dependent coagulation factor $\mathrm{X}$ of the blood coagulation cascade, which is considered a key factor in the activation of inflammation. A previous study reported that the levels of serum FXa in CFA-treated rats were significantly higher than those in control rats [29]. Moreover, Gang et al. demonstrated that F10 expression was elevated in patients with RA compared with normal subjects by using microarray datasets [30]. RA is an autoimmune disease characterized by inflammation of the synovial membrane. It is well known that the activation of the mitogen-activated protein kinase (MAPK) pathway is closely related to the inflammatory development of RA [31]. Meanwhile, F10 could boost MAPK activation to release chemokines and inflammatory cytokines [32]. F10 also has a proinflammatory action via protease-activated receptor 2 (PAR2) in many cell types [33]. It has been reported that FXa induces JAK2, STAT3 and MAPK phosphorylation through activation of PAR2, PDGF and IL-6, which may have a drastic role in RA progression [29]. Hence, we hypothesize that the reduction in F10 expression may be a target for combination drug therapy to alleviate RA.

SERPINA3, also called alpha-1-antichymotrypsin (ACT), is a member of the serine protease inhibitor family. It is believed that SERPINA3 contributes to the activation of proinflammatory cytokines, pathogen degradation and tissue remodeling via cathepsin $\mathrm{G}[34,35]$. SERPINA3 is a positive acute-phase reactant in the inflammatory process. When inflammation occurs, SERPINA3 is released into the circulation and inhibits the activities of various serine proteases [36]. In the early stage of RA, the level of SERPINA3 in serum is increased and positively correlated with the levels of CRP, ESR and morning stiffness, suggesting that it may be used as an additional marker for RA [37]. There are several limitations in this study. Due to the small sample size and significant individual differences, only a few DEPs were screened out. In a follow-up study, we will enroll more patients via a multicenter study and examine the details of these DEPs.

\section{Conclusions}

This proteomics study provides reference proteins for future research on RA treatment. The results of our differential protein expression analysis provide a preliminary mechanism for the combination of imrecoxib, MTX, LEF and TGP and help to improve RA through these mechanisms.

\section{Declarations}

\section{Conflicts of interest}

The authors have no conflict of interest to declare.

\section{Funding statement}


This work was supported by the Clinical Research Special Funds of Wu Jieping Medical Foundation (NO 320.6750.2020-03-6).

\section{Acknowledgements}

We thank the School of Life Sciences of USTC for technical support and thank all the patients who involved in the study.

\section{Author contribution}

Quan Xia contributed to the study conception and design. Material preparation and sample processing were performed by Haixia Yu and Xing Fang. Pathological diagnosis, collection of serum from patients were performed by Xuejing Ma and Chunlan Yang. Data analysis was performed by Tingting Wang and Lanxin Qin. The first draft of the manuscript was written by Haixia Yu and revised by Xianjun Fang. All authors commented on previous versions of the manuscript. All authors read and approved the final manuscript.

\section{References}

1. Toussirot E, Bonnefoy F, Vauchy C, Perruche S, Saas P (2021) Mini-Review: The Administration of Apoptotic Cells for Treating Rheumatoid Arthritis: Current Knowledge and Clinical Perspectives, Frontiers in immunology, 12630170

2. Lin YJ, Anzaghe M, Schulke S (2020) Update on the Pathomechanism, Diagnosis, and Treatment Options for Rheumatoid Arthritis, Cells, 9

3. Smolen JS, Breedveld FC, Burmester GR et al (2016) Treating rheumatoid arthritis to target: 2014 update of the recommendations of an international task force. Ann Rheum Dis 75:3-15

4. Gaffo A, Saag KG, Curtis JR (2006) Treatment of rheumatoid arthritis, American journal of healthsystem pharmacy: AJHP : official journal of the American Society of Health-System Pharmacists. 63:2451-2465

5. Smolen JS, Aletaha D, McInnes IB (2016) Rheumatoid arthritis. Lancet 388:2023-2038

6. Hazlewood GS, Barnabe C, Tomlinson G, Marshall D, Devoe D, Bombardier C (2016) Methotrexate monotherapy and methotrexate combination therapy with traditional and biologic disease modifying antirheumatic drugs for rheumatoid arthritis: abridged Cochrane systematic review and network meta-analysis. BMJ 353:i1777

7. Gomides APM, de Albuquerque CP, Santos ABV et al (2020) Rheumatoid artrhitis treatment in Brazil: data from a large real-life multicenter study. Adv Rheumatol 60:16

8. Hodkinson B, Magomero KR, Tikly M (2016) Combination leflunomide and methotrexate in refractory rheumatoid arthritis: a biologic sparing approach. Therapeutic Adv Musculoskelet disease 8:172179 
9. Liang C, Li J, Lu C et al (2019) HIF1alpha inhibition facilitates Leflunomide-AHR-CRP signaling to attenuate bone erosion in CRP-aberrant rheumatoid arthritis. Nat Commun 10:4579

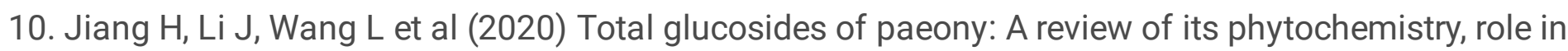
autoimmune diseases, and mechanisms of action. J Ethnopharmacol 258:112913

11. Chen Z, Li XP, Li ZJ, Xu L, Li XM (2013) Reduced hepatotoxicity by total glucosides of paeony in combination treatment with leflunomide and methotrexate for patients with active rheumatoid arthritis. Int Immunopharmacol 15:474-477

12. Liu Y, Zhang R, Li Z et al (2019) Lack of effect of Imrecoxib, an innovative and moderate COX-2 inhibitor, on pharmacokinetics and pharmacodynamics of warfarin in healthy volunteers. Sci Rep 9:15774

13. Huang $Y$, Wang $\mathrm{H}$, Chen $\mathrm{Z}$ et al (2019) Efficacy and safety of total glucosides of paeony combined with methotrexate and leflunomide for active rheumatoid arthritis: a meta-analysis, Drug design, development and therapy. 13:1969-1984

14. Teitsma XM, Jacobs JWG, Concepcion AN et al (2018) Explorative analyses of protein biomarkers in patients with early rheumatoid arthritis achieving sustained drug-free remission after treatment with tocilizumab- or methotrexate-based strategies: from transcriptomics to proteomics, Clinical and experimental rheumatology. 36:976-983

15. Sparks JA, Arthritis R (2019) Ann Intern Med 170:ITC1-ITC16

16. Si JB, Kim B, Kim JH (2021) Transthyretin Misfolding, A Fatal Structural Pathogenesis Mechanism,International journal of molecular sciences, 22

17. Vieira M, Saraiva MJ (2014) Transthyretin: a multifaceted protein. Biomol concepts 5:45-54

18. Lee J, Mun S, Kim D et al (2019) Proteomics Analysis for Verification of Rheumatoid Arthritis Biomarker Candidates Using Multiple Reaction Monitoring, Proteomics.Clinical applications, $13 \mathrm{e} 1800011$

19. Ni M, Wei W, Feng Q et al (2013) Transthyretin as a potential serological marker for the diagnosis of patients with early rheumatoid arthritis, Clinical and experimental rheumatology. 31:394-399

20. Phull AR, Nasir B, Haq IU, Kim SJ (2018) Oxidative stress, consequences and ROS mediated cellular signaling in rheumatoid arthritis, Chemico-biological interactions. 281:121-136

21. Mititelu RR, Padureanu R, Bacanoiu M Inflammatory and Oxidative Stress Markers-Mirror Tools in Rheumatoid Arthritis, Biomedicines, 8 et al (2020)

22. Lee CC, Ding X, Zhao T, Cells et al (2019) J Immunol 202:991-1002

23. Serada S, Fujimoto M, Ogata A et al (2010) iTRAQ-based proteomic identification of leucine-rich alpha-2 glycoprotein as a novel inflammatory biomarker in autoimmune diseases. Ann Rheum Dis 69:770-774

24. Fujimoto M, Serada S, Suzuki K et al (2015) Leucine-rich alpha2 -glycoprotein as a potential biomarker for joint inflammation during anti-interleukin-6 biologic therapy in rheumatoid arthritis. Arthritis \& rheumatology 67:2056-2060 
25. Wang N, Wu W, Qiang C et al (2021) Protective Role of Collectin 11 in a Mouse Model of Rheumatoid Arthritis. Arthritis \& rheumatology 73:1430-1440

26. Niu Q, Cai B, Huang ZC, Shi YY, Wang LL (2012) Disturbed Th17/Treg balance in patients with rheumatoid arthritis. Rheumatol Int 32:2731-2736

27. Urushima H, Fujimoto M, Mishima T et al (2017) Leucine-rich alpha 2 glycoprotein promotes Th17 differentiation and collagen-induced arthritis in mice through enhancement of TGF-beta-Smad2 signaling in naive helper T cells. Arthritis Res therapy 19:137

28. Wang X, Abraham S, McKenzie JAG et al (2013) LRG1 promotes angiogenesis by modulating endothelial TGF-beta signalling. Nature 499:306-311

29. El-Ghafar O, Helal GK, Abo-Youssef AM (2020) Apixaban exhibits anti-arthritic effects by inhibiting activated factor X-mediated JAK2/STAT3 and MAPK phosphorylation pathways. Inflammopharmacology 28:1253-1267

30. Gang X, Sun Y, Li F et al (2017) Identification of key genes associated with rheumatoid arthritis with bioinformatics approach. Medicine 96:e7673

31. Meng Y, Ji J, Xiao X et al (2021) Ononin induces cell apoptosis and reduces inflammation in rheumatoid arthritis fibroblast-like synoviocytes by alleviating MAPK and NF-kappaB signaling pathways. Acta Biochim Pol 68:239-245

32. Cirino G, Cicala C, Bucci M et al (1997) Factor Xa as an interface between coagulation and inflammation. Molecular mimicry of factor $\mathrm{Xa}$ association with effector cell protease receptor-1 induces acute inflammation in vivo. J Clin Investig 99:2446-2451

33. Matsuura T, Soeki T, Fukuda D et al (2021) Activated Factor X Signaling Pathway via ProteaseActivated Receptor 2 Is a Novel Therapeutic Target for Preventing Atrial Fibrillation. Circulation journal: official journal of the Japanese Circulation Society 85:1383-1391

34. Guzman-Herrera N, Perez-Najera VC, Salazar-Olivo LA (2021) Alpha-1-Antichymotrypsin: A Common Player for Type 2 Diabetes and Alzheimer's Disease. Curr Diabetes Rev 17:e121020186817

35. Gao S, Zhu H, Zuo X, Luo H, Cathepsin G (2018) Its Role in Inflammation and Autoimmune Diseases. Archives of rheumatology 33:498-504

36. Baker C, Belbin O, Kalsheker N, Morgan K (2007) SERPINA3 (aka alpha-1-antichymotrypsin), Frontiers in bioscience: a journal and virtual library. 12:2821-2835

37. Chard MD, Calvin J, Price CP, Cawston TE, Hazleman BL (1988) Serum alpha 1 antichymotrypsin concentration as a marker of disease activity in rheumatoid arthritis. Ann Rheum Dis 47:665-671

\section{Figures}


Figure 1

a

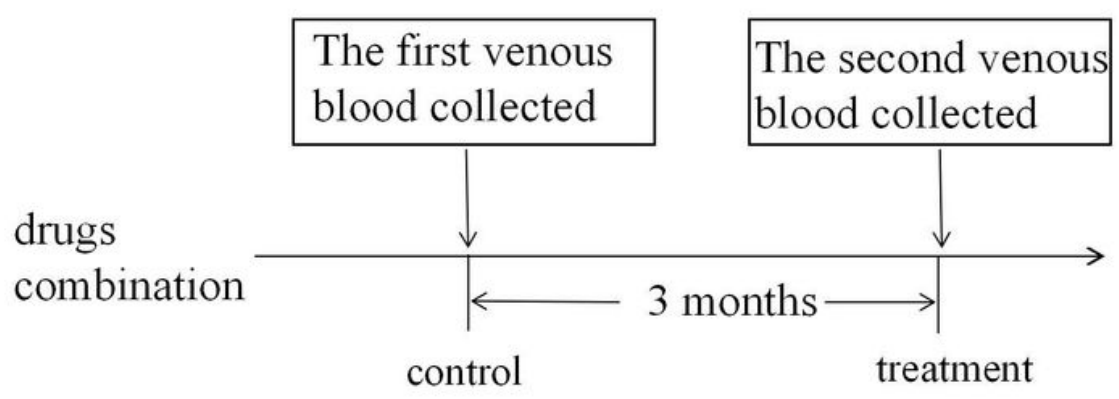

b

C
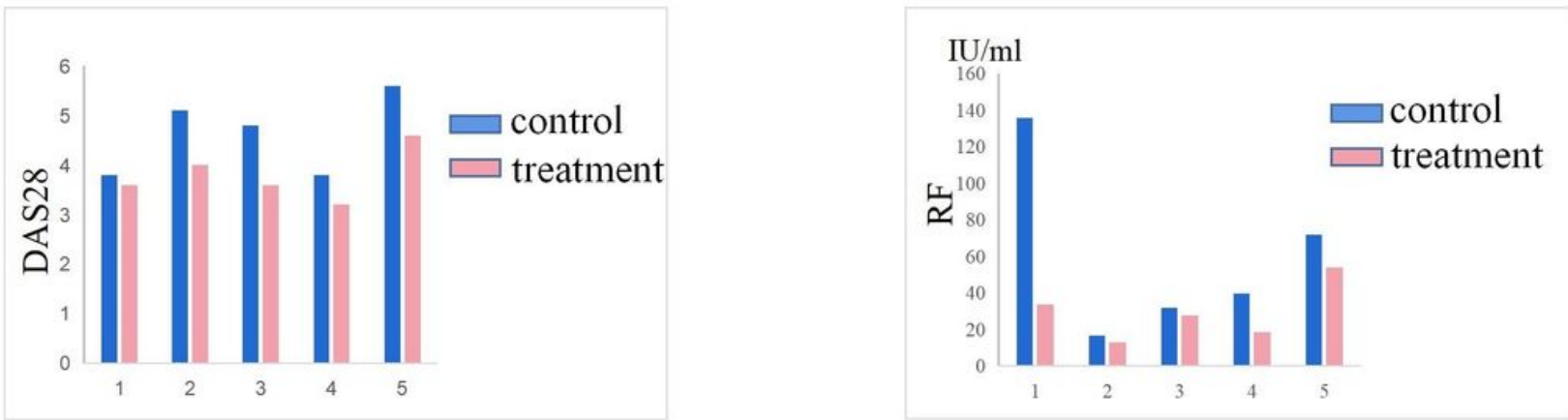

Figure 1

The treatment outcome of the combination therapy for RA. (A) The schematic of serum sampling. (B) DAS28 score of 5 patients before and after the combination treatment. (C) The RF values of 5 patients before and after the combination treatment. 
Figure 2

a

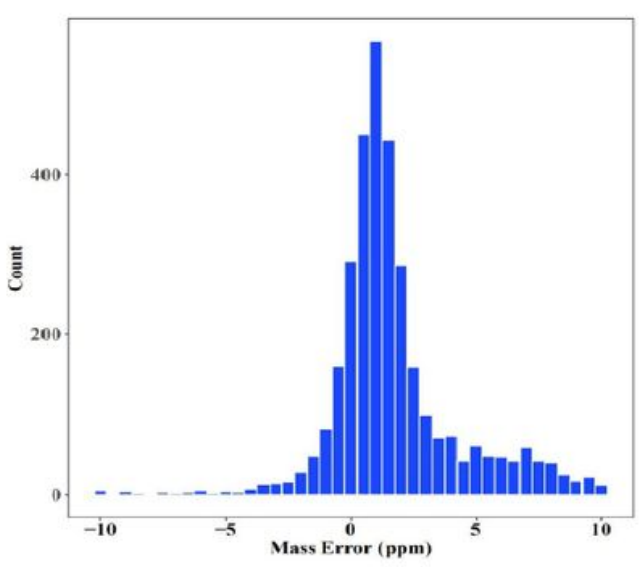

c

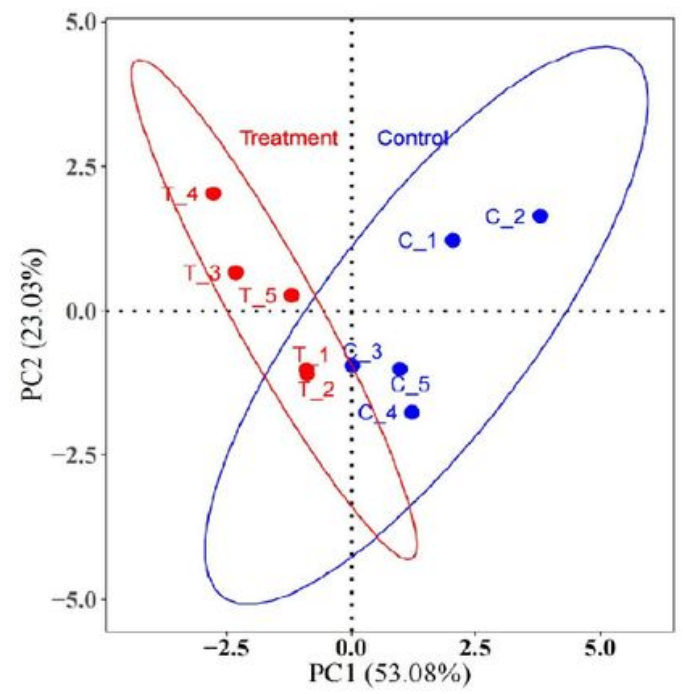

$\mathrm{b}$

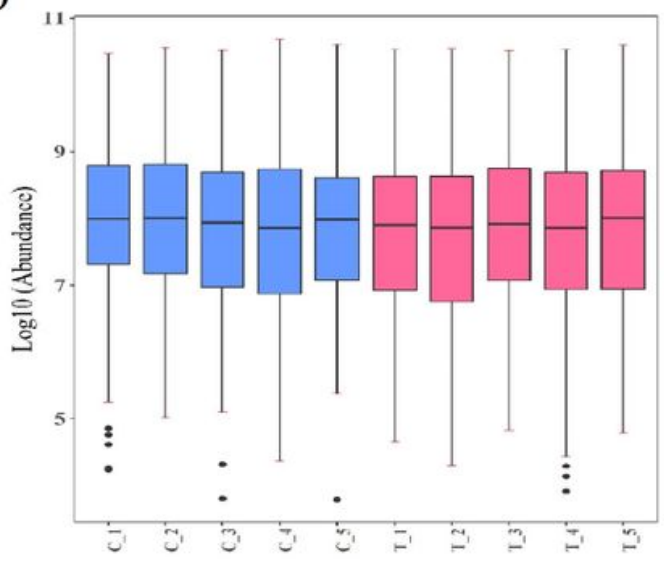

$\mathrm{d}$

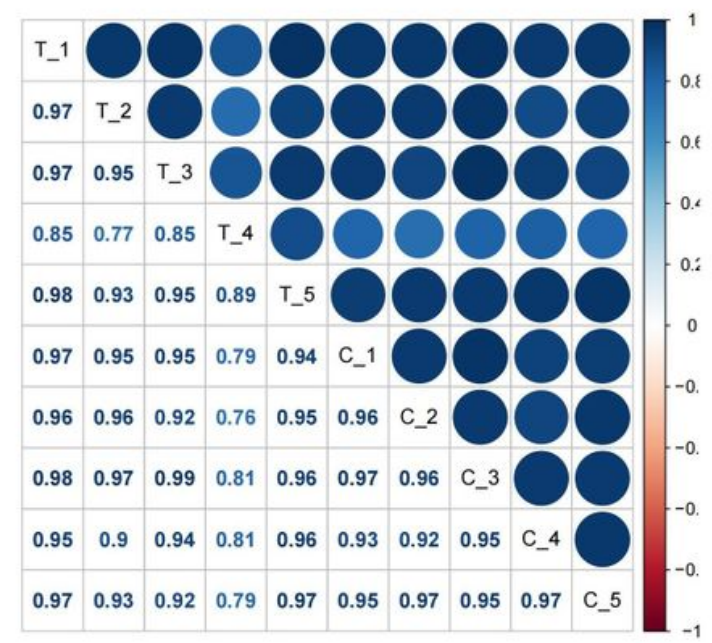

Figure 2

Quality control and unsupervised analysis of mass spectrometry data. (A) Mass error distribution of all identified peptides. The $Y$ axis represents the count of PSMs (peptide-spectrum matches), and the $X$ axis represents mass error. (B) Box-plot analysis of standardized protein abundance in all samples. The samples are presented on the horizontal axis, and the normalized amount of expression is shown on the vertical axis. (C) Principal component analysis. The data revealed two separate DEP clusters that can distinguish the samples taken before combination treatment from those taken after the treatment. (D) Analysis of the correlation coefficient between each sample. T indicates the serum of RA patients collected at the second visit after 3 months of combination drug therapy. $\mathrm{C}$ indicates the serum of RA patients collected at the first visit. 
Figure 3

a

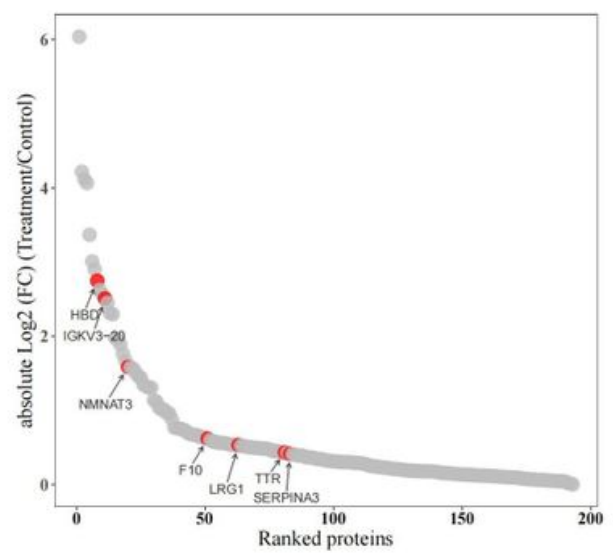

b

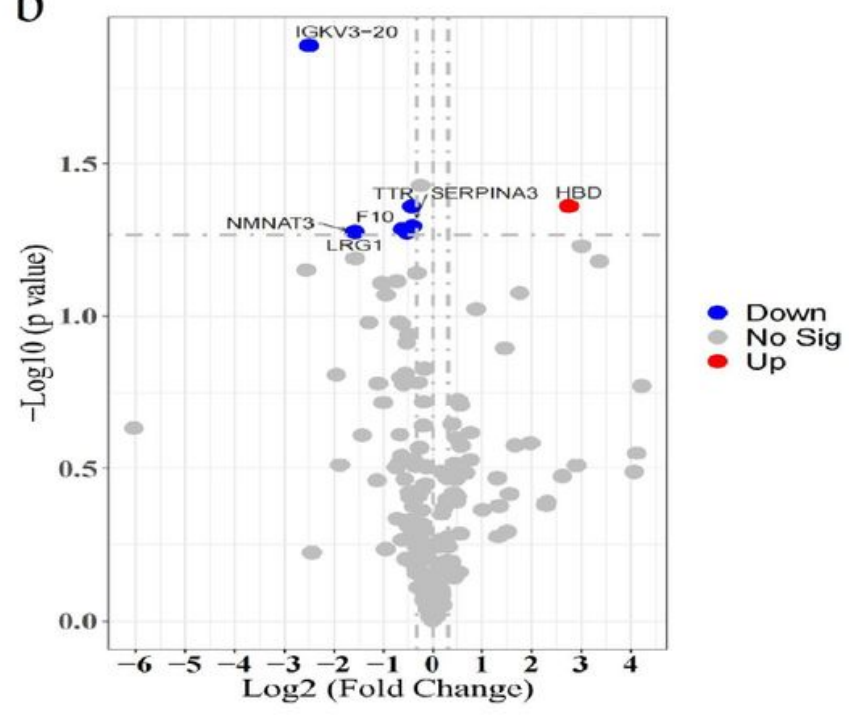

C

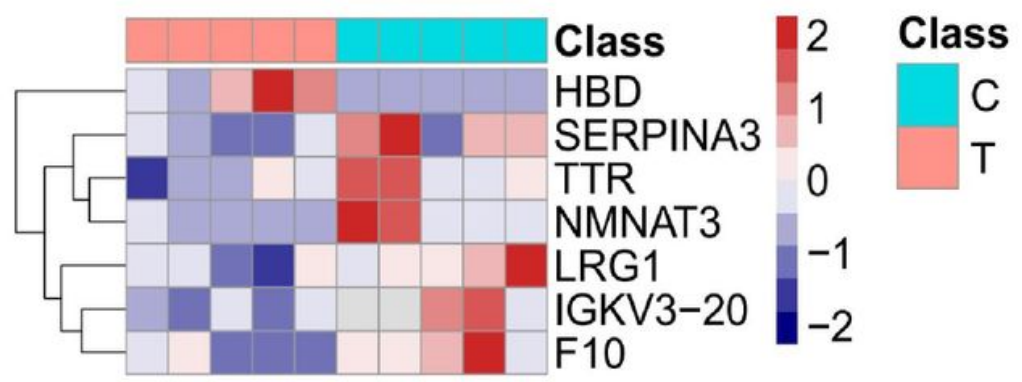

Figure 3

Identification of DEPs in serum. (A) Waterfall analysis of identified proteins. The fold changes of expression values for all identified proteins are log2 transformed, and the fold changes are absolute values. (B) Volcano plots of identified proteins. Red dots represent upregulated proteins, and blue dots represent downregulated proteins. (C) Hierarchical clustering heatmap showing the DEPs between the treatment and control groups. 
Figure 4
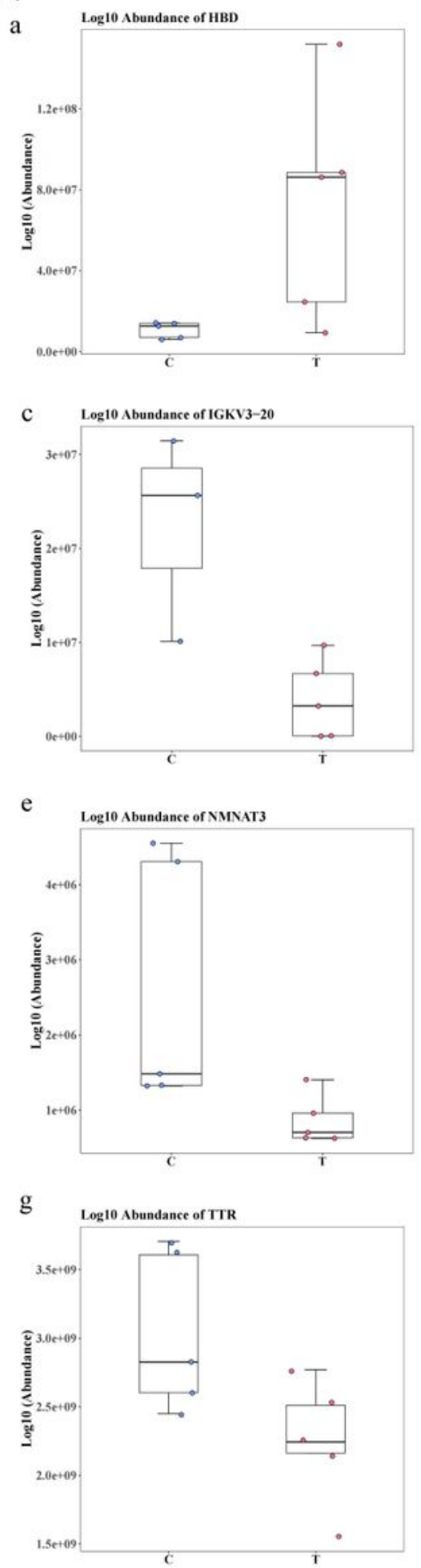
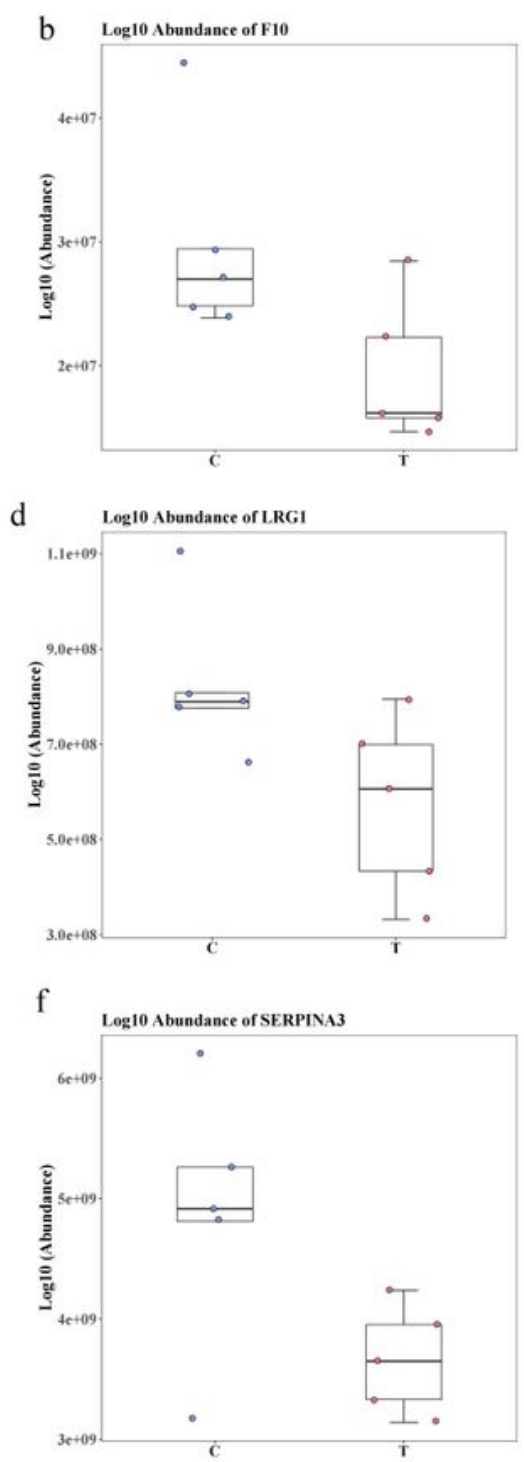

\section{Figure 4}

Abundance of DEPs in proteomics analysis. 
Figure $5 \mathrm{a}$

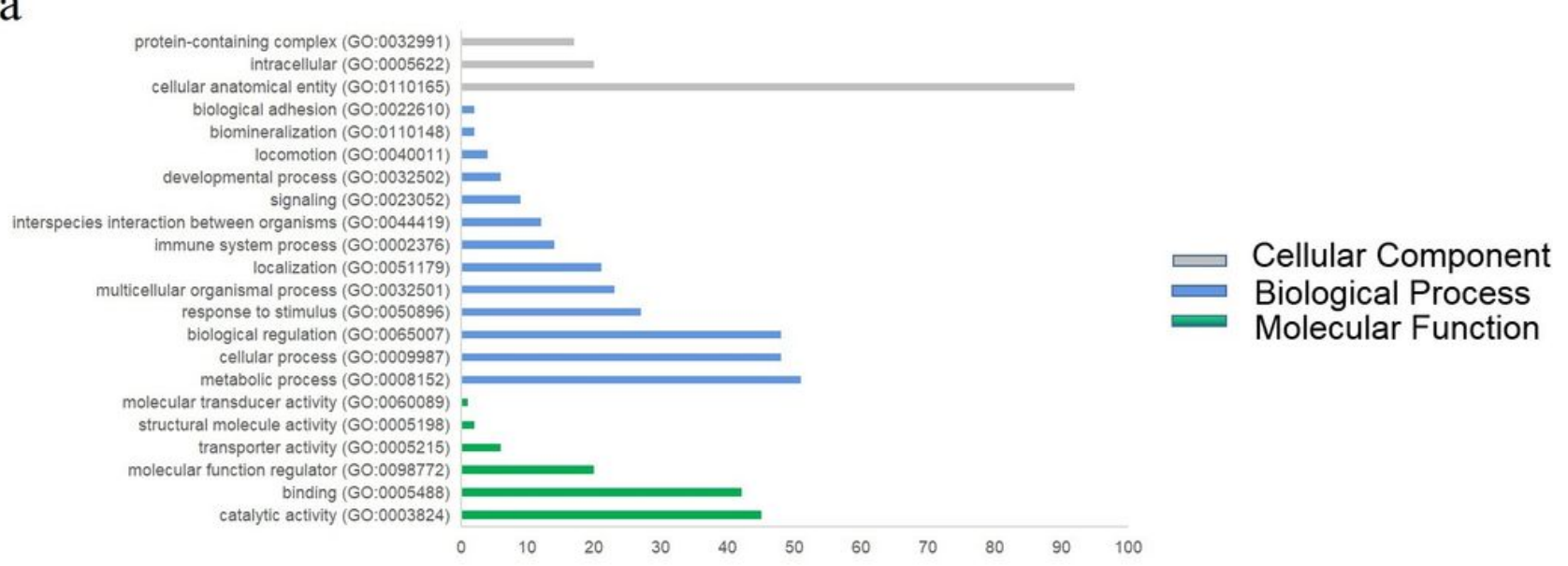

b

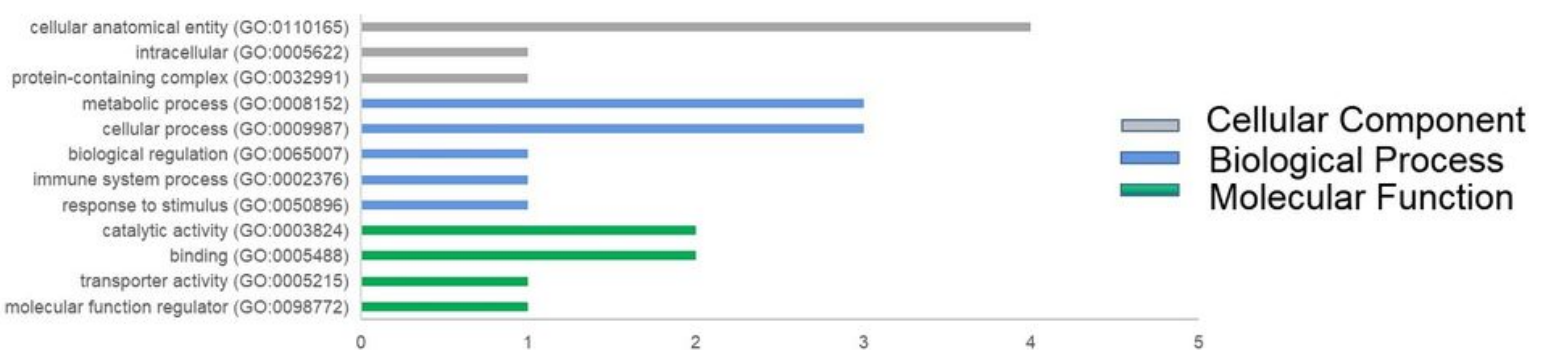

\section{Figure 5}

Functional GO classification analysis. (A) Functional GO classification of identified proteins. (B) Functional GO classification of DEPs. 